\title{
Spiers Memorial Lecture
}

\section{Molecular mechanics and molecular electronics}

\author{
Robert Beckman, ${ }^{a}$ Kris Beverly, ${ }^{a}$ Akram Boukai, ${ }^{a}$ Yuri Bunimovich, ${ }^{a}$ Jang Wook \\ Choi ${ }^{a}{ }^{a}$ Erica DeIonno, ${ }^{a}$ Johnny Green, ${ }^{a}$ Ezekiel Johnston-Halperin, ${ }^{a}$ Yi Luo, ${ }^{a}$ \\ Bonnie Sheriff, ${ }^{a}$ J. Fraser Stoddart ${ }^{b}$ and James R. Heath ${ }^{* a}$
}

\author{
Received 15th September 2005, Accepted 20th September 2005 \\ First published as an Advance Article on the web 5th October 2005 \\ DOI: $10.1039 / b 513148 k$
}

\begin{abstract}
We describe our research into building integrated molecular electronics circuitry for a diverse set of functions, and with a focus on the fundamental scientific issues that surround this project. In particular, we discuss experiments aimed at understanding the function of bistable [2]rotaxane molecular electronic switches by correlating the switching kinetics and ground state thermodynamic properties of those switches in various environments, ranging from the solution phase to a Langmuir monolayer of the switching molecules sandwiched between two electrodes. We discuss various devices, low bit-density memory circuits, and ultra-high density memory circuits that utilize the electrochemical switching characteristics of these molecules in conjunction with novel patterning methods. We also discuss interconnect schemes that are capable of bridging the micrometre to submicrometre length scales of conventional patterning approaches to the near-molecular length scales of the ultra-dense memory circuits. Finally, we discuss some of the challenges associated with fabricated ultra-dense molecular electronic integrated circuits.
\end{abstract}

\section{Introduction}

Several years ago we began a research program that was focused around the concept of building a molecular electronic computing machine. Our goals were several-fold, including minimization of energy consumption per bit operation, development of computational and memory architectures that are both defect-tolerant and amenable to chemical assembly, ${ }^{1}$ and the development of parallel manufacturing approaches that scale to molecular dimensions. The longest term goal is to construct integrated nanoelectronic circuits with multiple functions. These functions include the traditional electronic tasks of routing, logic and memory, but can also include non-traditional tasks such as biomolecular or chemical sensing, photonics, actuation, etc., that are enabled through molecular scale fabrication. Our 'vision' for such an integrated circuit is shown in Fig. 1. The crossbar architecture that is the dominant circuit theme shown in the figure is loosely based upon the Teramac supercomputer - a machine that we demonstrated could provide an excellent model system for nanoscale and molecular electronics. ${ }^{1}$

The challenges towards constructing such a multifunctional computational machine are nontrivial. One challenge surrounds the development of patterning techniques that can approach molecular dimensions in terms of both feature size and feature pitch. A second requires the development of architectures that allow for the bridging of length scales between the micrometre and sub-micrometre world of lithographic patterning to the nanometre scale world of self-assembly (the multiplexers of Fig. 1). A third challenge revolves around the development of molecular

\footnotetext{
${ }^{a}$ Caltech Chemistry, MC 127-72 1200 East California Boulevard, Pasadena CA 91125, USA.

E-mail:heath@caltech.edu

${ }^{\boldsymbol{b}}$ The California NanoSystems Institute and Department of Chemistry and Biochemistry, UCLA 405 Hilgard

Avenue, Los Angeles CA 90095-1569, USA.

E-mail: stoddart@chem.ucla.edu
} 


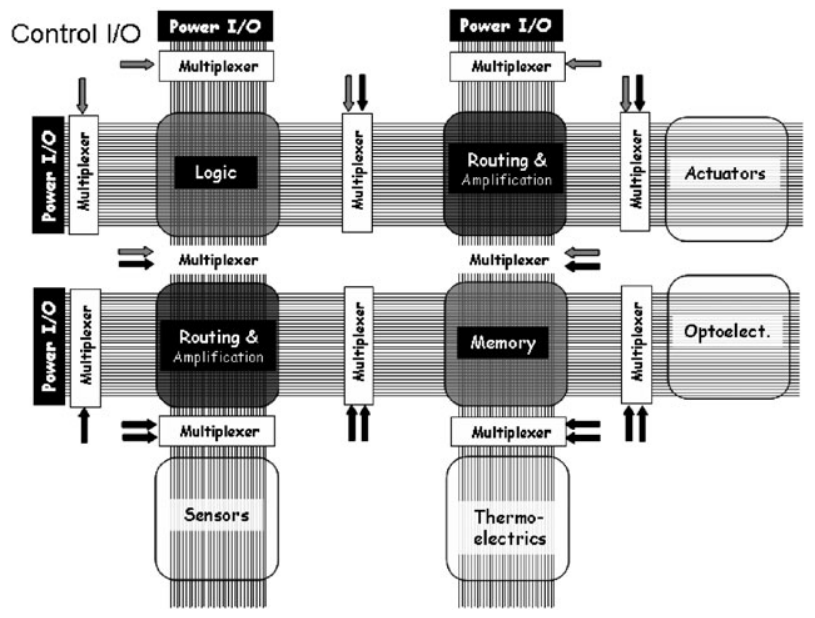

Fig. 1 A molecular and nanoelectronic computational platform based upon a patchwork of crossbar structures. Various traditional and non-traditional functions are illustrated, with multiplexers (and demultiplexers) controlling communication between the various functions and input/output (I/O) with the outside world. This particular circuit architecture is defect tolerant and amenable to both traditional and non-traditional patterning methods.

electronic switches. However, the fundamental scientific issues that must be addressed to meet each of these challenges are very rich. In this paper, we will discuss some of our work in these various areas, with a focus on both the fundamental scientific problems that have been addressed, and the outstanding questions that must still be answered.

Note that although many applications are labeled in the Fig. 1 circuit diagram, there is only one architecture - that of the crossbar-for all functions. The major hardware components that differentiate these various functions are the nanowire materials and the molecules that are coated on top of the nanowires or sandwiched in between the junctions. For the memory applications, the molecular systems that we have explored have been bistable molecular mechanical systems known as electrochemically switchable donor-acceptor [2]rotaxanes and [2]catenanes. It is towards these molecules, and the associated molecular electronic devices, that we first turn our attention.

\section{Molecular mechanical switches}

Over the past several years two previously distinct areas of chemical research - the synthesis of molecular mechanical systems, and the fabrication of solid-state molecular electronics devices ${ }^{2}$ and circuits - have been conjoined. The resulting union has led to the scientific demonstration of a number of interesting devices, including molecular electronic switches, ${ }^{3}$ light harvesting devices, ${ }^{4}$ molecular electronic based random access memory and configurable logic circuits, ${ }^{5}$ molecular mechanical biosensors, ${ }^{6}$ actuated molecular valves, ion channel mimics, molecular muscles, ${ }^{7}$ and novel electrochromic devices. ${ }^{8}$ Equally interesting has been the emerging picture of large-amplitude molecular actuation and how it is influenced and modified by the surrounding physical and chemical environments. ${ }^{9}$

In Fig. 2 we present the molecular structure of a bistable [2]rotaxane $\left(\mathrm{RATTF}^{4+}\right)$ molecular switch, along with a potential energy diagram describing the bistable nature of the switch. This bistable [2]rotaxane is composed of $\pi$-electron-accepting cyclobis(paraquat- $p$-phenylene) $\left(\mathrm{CBPQT}^{4+}\right)$ ring (blue) that encircles either a tetrathiafulvalene (TTF) unit (green) or a 1,5dioxynapthalene (DNP) unit (red), both $\pi$-electron-donating systems. These mechanically-interlocked molecular compounds and other closely related bistable rotaxanes ${ }^{10}$ as well as rotaxanes constructed from different donor-acceptor units ${ }^{11}$ and from hydrogen-bonded systems ${ }^{12}$ and transition metal templates, ${ }^{13}$ have been investigated in depth previously. Under ambient conditions in acetonitrile solution, the CBPQT ${ }^{4+}$ ring in RATTF $^{4+}$ encircles the TTF unit preferentially 

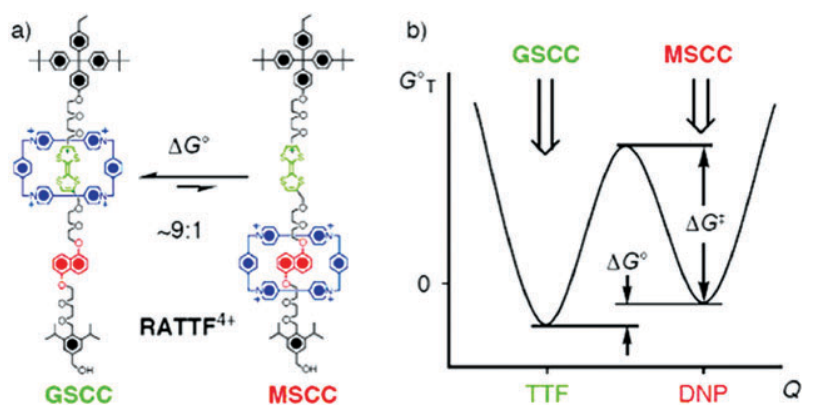

Fig. 2 (a) Structural formulas of the two translational isomers of the bistable rotaxane RATTF ${ }^{4+}$ correspond- $^{-}$ ing to the ground state co-conformation (GSCC) and the metastable state co-conformation (MSCC). (b) Potential energy surface for the bistable RATTF ${ }^{4+}$ where the energy wells correspond to the GSCC and MSCC. The free energy difference $\Delta G^{\circ}$, between the wells and the free energy barrier to relaxation, $\Delta G^{\ddagger}$, from the MSCC to the GSCC are defined against a normal coordinate, $Q$, representing translation of the ring along the dumbbell component of the [2]rotaxane.

( $>90 \%$ ) with respect to the DNP unit. This equilibrium is described by the $\Delta G_{298}{ }^{\circ}$ change shown in Fig. $2 \mathrm{~b}$ where $\Delta G^{\circ}=+1.6 \mathrm{kcal} \mathrm{mol}^{-1}$ when the $\mathrm{CBPQT}^{4+}$ ring moves from the TTF to the DNP unit. Hence, the co-conformation $(\mathrm{CC})$ with the $\mathrm{CBPQT}^{4+}$ ring encircling the TTF is unit is referred to as the ground state co-conformation (GSCC). The first two oxidation states of RATTF ${ }^{4+}$ correspond to the $\mathrm{TTF}^{0} \rightarrow \mathrm{TTF}^{\bullet+} \rightarrow \mathrm{TTF}^{2+}$ processes. Upon formation of $\mathrm{TTF}^{\bullet+}$ cation radical, the Coulombic repulsion between the $\mathrm{CBPQT}^{4+}$ ring and the $\mathrm{TTF}^{\cdot+}$ results ${ }^{14,15}$ in translation of the ring to the DNP unit. This process is fast (on the millisecond time scale) and is believed to convert all of the GSCC into the MSCC. When the TTF ${ }^{*}$ cation radical is reduced back to $\mathrm{TTF}^{0}$, the $\mathrm{CBPQT}^{4+}$ ring remains around the DNP unit for a period of time. This translational isomer of the GSCC is the metastable state co-conformation (MSCC). Recovery of the MSCC/GSCC equilibrium distribution $(\sim 1: 9)$ is an activated process. This switching cycle can be detected by a number of experimental observations. First, the lowest oxidation potential (corresponding to $\mathrm{TTF}^{0} \rightarrow \mathrm{TTF}^{\cdot+}$ ) of the GSCC is $+490 \mathrm{mV}$, while that for the MSCC is $+310 \mathrm{mV}$. (All potentials referenced to an $\mathrm{Ag} / \mathrm{AgCl}$ electrode.) Second, the colors of GSCC- and MSCC-dominated solutions are green and red, respectively. Thus, electrochemistry and spectroscopy can be employed to quantify the MSCC/GSCC ratio in such a bistable rotaxane at any given time. Third, the (activated) relaxation of an MSCC- back to a GSCC-dominated distribution is temperature-dependent and so the kinetic parameters may be quantified through time- and temperature-dependent measurements. For example, the $\Delta G_{298}{ }^{\ddagger}$ for this process in the case ${ }^{16}$ of $\operatorname{RATTF}^{4+}$ is $16.2( \pm 0.3) \mathrm{kcal} \mathrm{mol}^{-1}$.

Consider, for example, a molecule that, when incorporated into an electronic device, acts as a solid-state molecular resistor ${ }^{17,18}$ or rectifier, ${ }^{19-23}$ or perhaps generates some unusual device characteristic, such as negative differential resistance. ${ }^{24,25}$ Such properties are uniquely studied in the device setting and may arise from a distinctly molecular property, or from the result of the molecule/electrode interaction. When these molecules are studied in the liquid solution phase, those same properties are no longer directly revealed in experiments, although donor-acceptor charge transfer spectroscopy can provide an indirect probe of molecular rectification. In other words, certain properties arise through a combination of the molecular design and the physical (device) environment in which that molecule is incorporated. Molecular mechanical motion, however, has certain key characteristics that are experimentally observable and that are independent of environment. This is especially so for the case of the synthetically flexible catenanes and rotaxanes highlighted in this Discussion. ${ }^{26}$ There are not only multiple ways to design and control molecular mechanical motion in these systems, but there are also a variety of experimental methods that can be employed to interrogate it, regardless of environment.

Our approach towards understanding switching within molecular electronic device settings has been to quantify how the environment of the molecular switch impacts the kinetic and thermodynamic parameters associated with electrochemically driven, bistable molecular mechanical switching. We have established two criteria that define a good molecular switch. First, the thermodynamics and kinetics of the molecular mechanical switching processes should correlate 

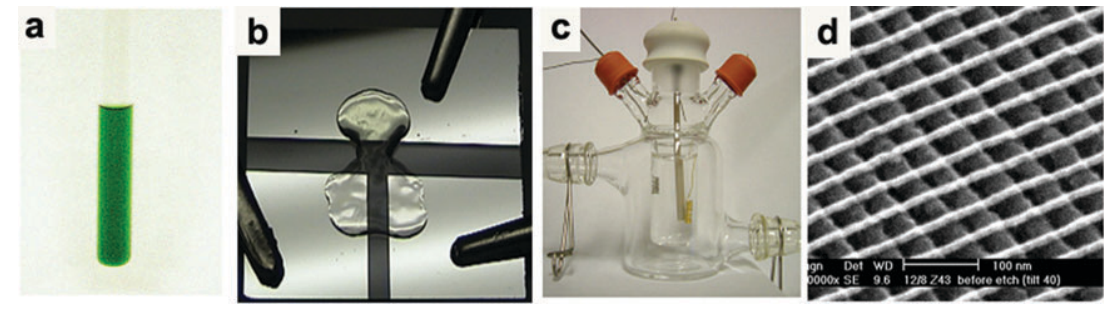

Fig. 3 The four physical environments in which rotaxanes and catenanes are discussed within this Lecture. (a) In organic solvent. (b) Solid state polymer electrolytes. The image shown here is of a three-terminal electrochemical cell that utilizes the switching of [2]rotaxanes and [2]catenanes as the basis of an electrochromic device. (c) Self-assembled monolayers on the surface of a working electrode. The example shown here is of a [2]rotaxane assembled on the surface an a working Au electrode within an electrochemical cell. (d) A molecular switch tunnel junction. Shown is a crosspoint memory in which a Langmuir monolayer of [2] rotaxanes is sandwiched between a top $\mathrm{Ti} / \mathrm{Al}$ electrode and a bottom poly-Si electrode.

to those same properties when measured in the solution phase. Second, control molecules shouldn't switch. The change between the solution phase and the sterically restricted environment of a molecular electronic device is likely to significantly modify at least some subset of the thermodynamic and kinetic parameters for switching. Thus, our approach has been to investigate bistable switching in [2] rotaxanes and [2]catenanes in a host of environments, ${ }^{27}$ including the solution phase, solid-state polymer electrolyte gels, self-assembled molecular monolayers tethered to the surface of working electrodes within electrochemical cells, ${ }^{15}$ and molecular switch tunnel junction devices. Representative images of each of these environments are shown in Fig. 3.

Much of our work on quantitating the switching kinetics and the equilibrium thermodynamics in these various environments has been published elsewhere. From those measurements, the mechanistic picture illustrated in Fig. 4 has emerged as being universal for both [2]catenanes and [2]rotaxanes across all physical environments. We initially discuss the switching kinetics (e.g. measurements of $\Delta G^{\ddagger}$ and other associated parameters from Fig. 2), starting with a self-assembled monolayer of bistable [2]rotaxanes on the surface of a working Au electrode. ${ }^{15}$ The objectives of these measurements are to quantify the recovery of the ground state equilibrium distribution (described by $\Delta G^{\circ}$ of Fig. 2 with an equilibrium constant of $K_{(\mathrm{D} / \mathrm{T})}{ }^{4+}$ as shown in Fig. 4) following electrochemical switching of a GSCC-dominated population (ground state) to an MSCC-dominated population (metastable state) of [2]rotaxanes.

Cyclic voltammetry (CV) scans, and accompanying descriptions of the redox processes, are shown in Fig. 5 for the surface-tethered [2]rotaxane and its dumbbell component.

In Fig. 6 we present two CV scans, taken in rapid succession, of just the reversible oxidization of the TTF groups on the same surface-tethered bistable [2]rotaxane.

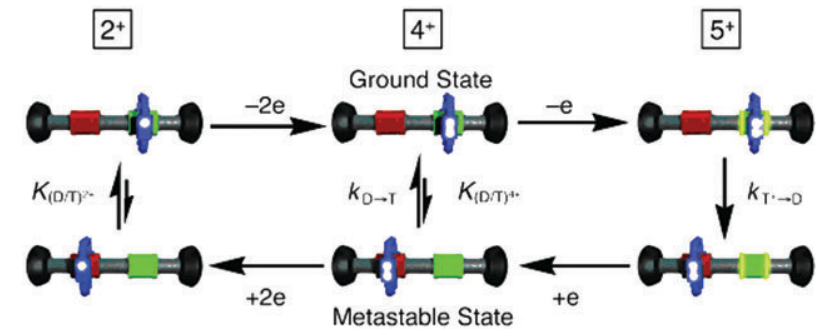

Fig. 4 The switching cycle of a bistable [2]rotaxane, starting with the ground state conformer. The green and red sites refer to the TTF and DN recognition units. When the TTF is oxidized, it is drawn as a highlighted green unit. The $\mathrm{CBPQT}^{4+}$ ring is modeled as the blue ring encircling the green site, with positive charges indicated as white spots. The metastable and ground state co-conformers are isoelectronic. The various rate constants indicated are discussed in the text. The charge states labeled mid-line in the drawing refer to the rotaxane structures below the labels. An exactly analogous mechanism fits the switching of bistable [2]catenanes that are constructed from similar host/guest components. 


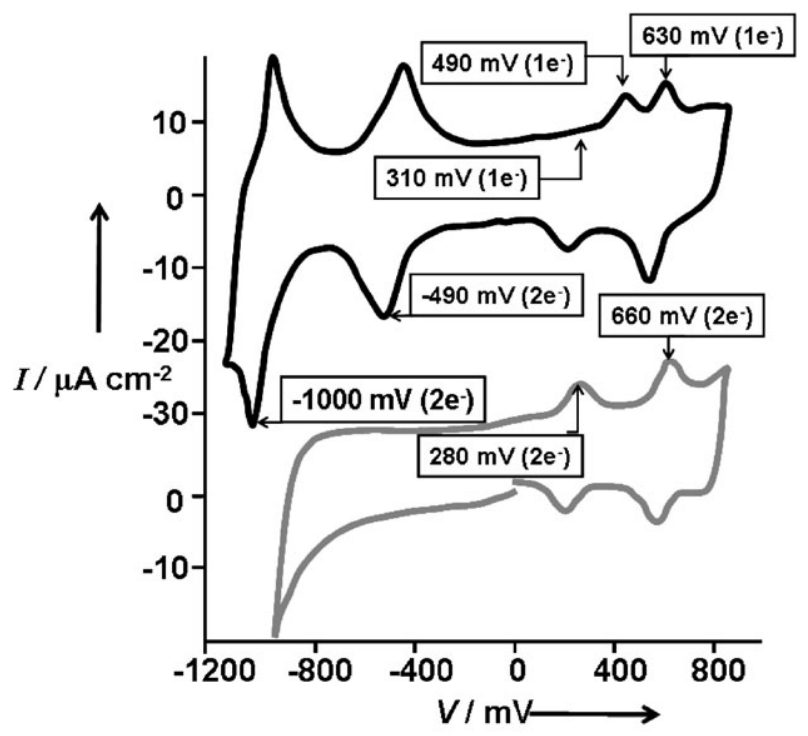

Fig. 5 Cyclic voltammograms (CVs) of a bistable [2]rotaxane tethered (through a disulfide linkage) to the surface of an $\mathrm{Au}$ working electrode within an electrochemical cell. All voltages are referenced to $\mathrm{Ag} / \mathrm{AgCl}$. The top CV trace is of a bistable [2]rotaxane containing TTF and DN recognition groups. In the ground state conformation, a CBQT $^{4+}$ ring preferentially encircles the TTF group. The two oxidation peaks at 490 and 630 $\mathrm{mV}$ correspond to $\mathrm{TTF}^{0} \rightarrow \mathrm{TTF}^{+}{ }^{+} \rightarrow \mathrm{TTF}^{2+}$ oxidation steps, which are reversed on the return sweep. The two reduction peaks at -490 and $-1000 \mathrm{mV}$ each correspond to two-electron processes, and are the CBQT ${ }^{4+} \rightarrow$ $\mathrm{CBQT}^{2+} \rightarrow \mathrm{CBQT}^{0}$ reduction processes. The bottom trace is a $\mathrm{CV}$ of the dumbbell component of the tethered [2] rotaxane (e.g. no $\mathrm{CBQT}^{4+}$ ring). Note that the $\mathrm{TTF}^{0} \rightarrow \mathrm{TTF}^{*}+$ process is shifted by $-210 \mathrm{mV}$, although the $\mathrm{TTF}^{\cdot+} \rightarrow \mathrm{TTF}^{2+}$ process is relatively unchanged. This indicates that the $\mathrm{CBQT}^{4+}$ ring translates to the DN site after the TTF group is singly oxidized.

Note that the first oxidation peak is observed to shift by $\sim-200 \mathrm{mV}$ between the first and second scans. This hysteresis implies that the $\mathrm{CBQT}^{4+}$ ring encircles the TTF-site during the first scan, but it has not yet returned to the TTF site when the second cv scan was carried out-i.e. it is still on the $\mathrm{DN}$ site. If a long time period existed between the first and second scans, then the two scans are observed to overlay (no hysteresis is observed in the first oxidation peak). This is because the ground state equilibrium distribution would have been re-established. Thus, this type of CV data can be measured as a function of temperature and time, and the kinetic parameters associated with the recovery of the GSCC-dominated equilibrium from a MSCC-dominated monolayer can be established. In a similar way, solution-phase data and data within polymer electrolytes can be collected. For all of these environments, the relative GSCC and MSCC confirmations can be established quantitatively. For molecular switch tunnel junction devices, the corresponding experiment is to electronically switch the device from the low- to the high-conducting state, and then follow the time-dependent decay back to the low-conducting state.

Such measurements can be carried out as a function of temperature. In this way, Eyring plots can be constructed for similar molecules in all four environments, and such plots and a table of the derived kinetic parameters are presented in Fig. 7. These type of measurements have been done for several [2]rotaxanes and [2]catenanes, and the picture that has emerged is that the switching (relaxation) kinetics are strongly dependent upon both the molecular structure of the switch, and the physical environment that the switch has been placed in. In fact, both effects can be harnessed to yield approximately equal kinetics control. For example, relaxation times in polymer-based electrochromic devices can be controlled over a dynamic range of approximately $10^{4}$ through control over the molecular structure of the switch. ${ }^{8}$ Similarly, taking an identical switch (with adaptations to the stopper groups that allow for it to be incorporated into different environments), the relaxation times are reduced by approximately $10^{4}$ or $10^{5}$ when moving from the solution phase to the polymer to the self-assembled monolayer to the molecular switch tunnel junction device. 


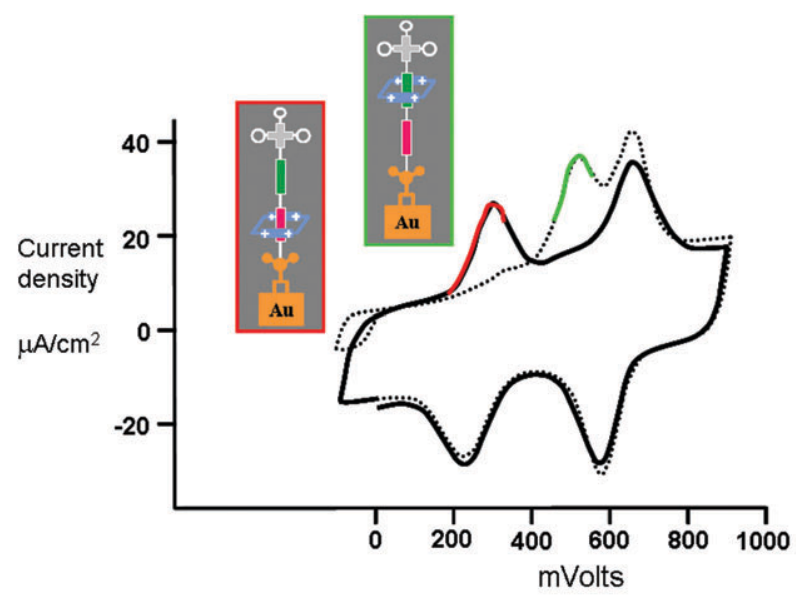

Fig. 6 Two cv scans taken in quick succession, following the TTF-oxidation process of the tethered bistable [2]rotaxane on the Au working electrode surface. The $\mathrm{TTF}^{0} \rightarrow \mathrm{TTF}^{\bullet+}$ process exhibits a hysteretic response between the first scan (dashed line) and the second scan (solid line). By integrating these two peaks (green and red) the relative concentrations of the MSCC and GSCC may be evaluated as a function of time and temperature, thereby allowing for a quantitative analysis of $\Delta G^{\ddagger}$ and associated kinetic parameters.

We have also measured the equilibrium thermodynamics within a wide variety of environments, and that work will appear in press soon. ${ }^{9 b}$ Briefly, we designed three molecules based upon differential scanning calorimetry measurements of the various host-guest complexes. One molecule $\left(\mathrm{RTTF}^{4+}\right)$ utilized the same [2] rotaxane switching motif shown in Fig. 2, but the hydrophilic stopper was replaced with a much bulkier group that contained three polyethylene glycol oligomers. For the second [2] rotaxane $\left(\mathrm{RBPTTF}^{4+}\right)$, we replaced the TTF with a bispyrrolotetrathiafulvalene (BPTTF) unit. A third control [2] rotaxane $\left(\mathrm{RBLOCK}^{4+}\right)$ was designed with an ethyl group at the base of the TTF group, and this served to lock the $\mathrm{CBQT}^{4+}$ ring so that it could not translate away from the TTF site, whether or not the TTF site was oxidized. $\mathrm{RTTF}^{4+}$ was designed to exhibit a temperature-independent ground state equilibrium that is dominated $(90 \%)$ by the $\mathrm{CBQT}^{4+}$ ring encircling the TTF site. RBPTTF $^{4+}$ exhibits a strong temperature dependent equilibrium that is

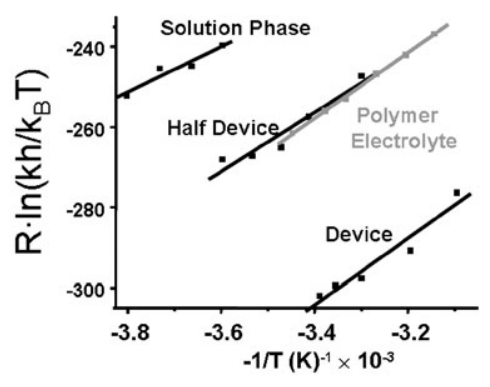

$\begin{array}{lrrrc}\text { DATA } & \mathrm{E}_{\mathrm{a}}\left(\mathrm{kcal} \mathrm{mol}^{-1}\right) & \Delta \mathrm{G}^{ \pm}\left(\mathrm{kcal} \mathrm{mol}^{-1}\right) & \Delta \mathrm{H}^{ \pm}\left(\mathrm{kcal} \mathrm{mol}^{-1}\right) & \Delta \mathrm{S}^{ \pm}\left(\mathrm{cal} \mathrm{mol}^{-1} \mathrm{~K}^{-1}\right) \\ \text { Solution: } & 14.1( \pm 1.2) & 15.9( \pm 1.2) & 13.5( \pm 1.2) & -8.9( \pm 5) \\ \text { SAM 1/2 device: } & 17.7( \pm 0.6) & 18.0( \pm 0.7) & 17.6( \pm 0.6) & -1.4( \pm 3) \\ \text { Polymer: } & 19.6( \pm 0.2) & 18.1( \pm 0.2) & 19.0( \pm 0.2) & 3.0( \pm 1.5) \\ \text { Tunnel Junction: } & 19.8( \pm 0.3) & 21.4( \pm 0.3) & 19.8( \pm 0.3) & 5.5( \pm 2)\end{array}$

Fig. 7 Eyring plots for bistable [2]rotaxane molecular switches entrained in four different environments as labeled on the graph. Kinetic parameters extracted from this and similar data are presented in the table. The [2]rotaxane contained TTF and DN recognition units and a $\mathrm{CBQT}^{4+}$ ring, but each molecule differed slighted in the terminal stoppers on the dumbbell component. The stoppers were modified to allow for adapting the molecule into the different physical environments. 


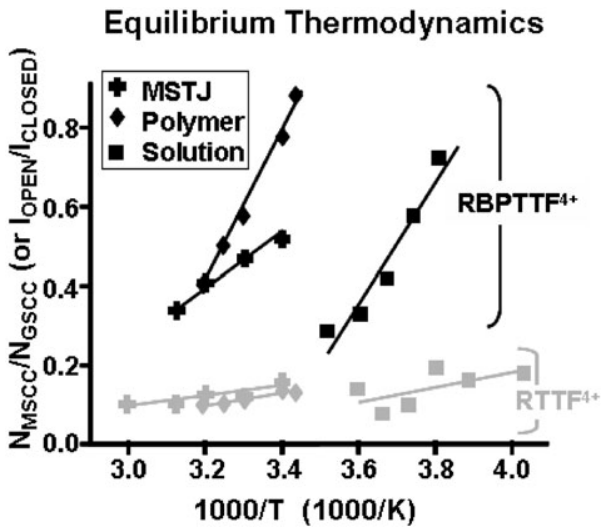

Fig. 8 The temperature-dependent GSCC/MSCC equilibria for three environments are presented for RBPTTF $^{4+}$ and RTTF $^{4+}$. Note that the large (entropically-driven) temperature dependence for RBPTTF ${ }^{4+}$, and the relative temperature independence of $\mathrm{RTTF}^{4+}$, is reflected in all three environments.

dominated by very different entropies when the $\mathrm{CBQT}^{4+}$ ring encircles the RBPTTF site $(-3.6$ cal $\left.\mathrm{K}^{-1} \mathrm{~mol}^{-1}\right)$ or the DN site $\left(-30.8 \mathrm{cal} \mathrm{K}^{-1} \mathrm{~mol}^{-1}\right)$. The entropy associated with the $\mathrm{CBQT}^{4+}$ ring encircling the TTF site was $\left(-22.1 \mathrm{cal} \mathrm{K}^{-1} \mathrm{~mol}^{-1}\right)$.

The high entropy values are ascribed to configurational entropy that arises from interactions between the $\mathrm{CBQT}^{4+}$ ring and the ethylene glycol bridging units. For these measurements, we quantified the amount of GSCC versus MSCC for the solution and polymeric environments using cyclic voltammetry. For the molecular switch tunnel junction environment, we evaluated the temperature dependent switching amplitude. Since the high conductance state is associated with the MSCC, a large ground state equilibrium population of the MSCC will reduce the switching amplitude.

RBLOCK $^{4+}$ was observed to be a poor switch in all environments, as expected. Data for RBPTTF $^{4+}$ and RATTF ${ }^{4+}$ are presented together in Fig. 8. This data provides strong evidence that the ground state equilibrium thermodynamics of these molecules is dominated by molecular structure, with the physical environment of the molecular switch playing only a small role.

\section{Molecular electronic circuits}

One of our motivations for developing molecular switches such as the RTTF $^{4+}$ molecule described above was to be able to apply them as the active (switching) devices within molecular electronic memory circuits. These switches hold a number of advantages (and disadvantages) over more traditional two-terminal switches, such as ferroelectric materials. First, since the switching property is distinctly molecular, devices based upon these switches should scale to near molecular dimensions without a significant change in the switching characteristics. This is distinct from solid state materials such as ferroelectrics. In those materials, a crystallographic domain is 'poled' using an electric field applied between the two junctions. For very small junction sizes, and thus small domains, the hysteretic response of the material vanishes. For magnetic materials, this is referred to as superparamagnetism. The second advantage is that the voltage at which the switches are opened or closed is quite stable, since the switching mechanism is electrochemical in nature (e.g. current has to flow in and out of molecular orbitals). For field poled devices, the actual poling process is driven by nucleation, and so is intrinsically statistical. This means that the voltage at which a ferroelectric bit switches can vary from junction to junction, or within a single junction, it varies from cycle to cycle. The net result is that, within a crosspoint memory architecture, it is difficult to get reliable performance from solid-state materials. Our molecular electronic switches, however, work well within a crosspoint environment. A more detailed discussion of this important issue can be found elsewhere. ${ }^{5}$ 


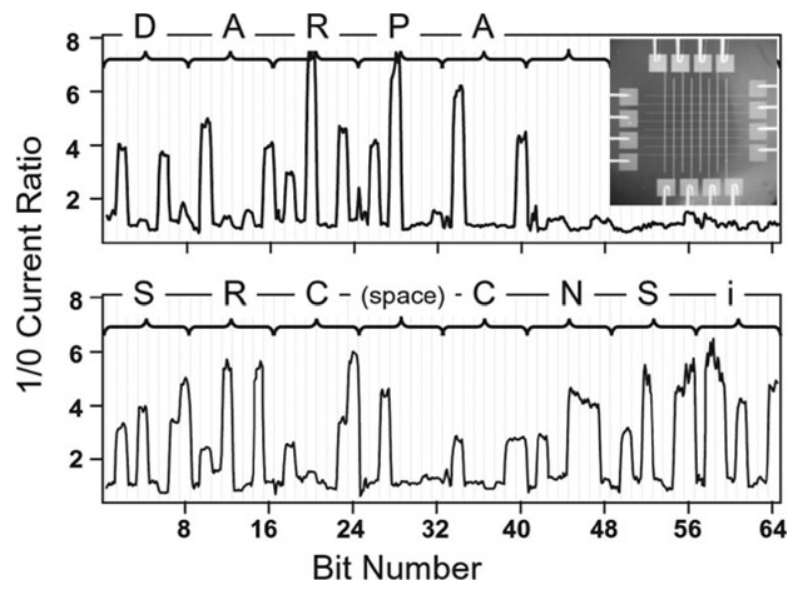

Fig. 9 The performance of a 64-bit molecular electronic crosspoint memory circuit (circuit shown in the upper right optical micrograph). The switching elements were bistable [2]rotaxanes, and the ASCII characters of the text shown above the two traces were stored as 1's and 0's, and then read out.

These molecular mechanical switches are not without their disadvantages. The full switching mechanism contains thermally activated processes, which means that the devices will exhibit temperature-dependent variations in their operation. ${ }^{9 b}$ Second, molecular switch tunnel junction devices (MSTJs) made from [2]rotaxanes and [2]catenanes have, to date, not been particularly robust performers. For example, a switch that may be cycled 1000 times is, at present, a pretty good switch. Finally, the switching mechanism relies upon high-amplitude molecular mechanical motion, and so it is fairly slow. For different bistable [2]rotaxanes, Willner's group has quantified some of the solid-state kinetic processes and has found that the step that corresponds to $\mathrm{kT}^{+} \rightarrow \mathrm{D}$ occurs on the millisecond time scale. ${ }^{28}$ Such slow switching speeds, however, are not a particularly serious limitation when compared to the other two issues, since parallel architectures (of which the crossbar is one) generate speed through circuit design, rather than through device switching speed.

A few years ago we reported on a 64-bit molecular electronic memory circuit (Fig. 10). ${ }^{5}$ That circuit utilized bistable [2]rotaxanes, formed as a Langmuir-Blodgett (LB) monolayer. The LB film was sandwiched between a bottom n-type poly-silicon electrode and a top aluminium electrode. Between the $\mathrm{Al}$ electrode and the molecular film was deposited a thin $(5 \mathrm{~nm}) \mathrm{Ti}$ adhesion layer. Using this memory circuit, small data strings could be stored, read out, erased, and then new information could be rewritten into the memory. This memory circuit, considering it was the first of its kind, performed reasonably well.
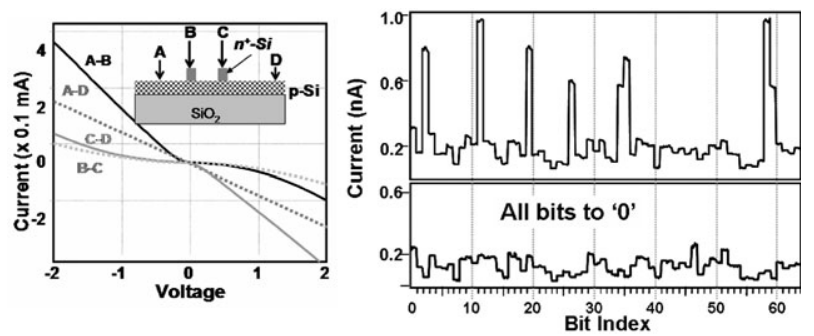

Fig. 10 A molecular electronic memory circuit in which diodes are constructed at each of the crosspoint junctions by establishing a $\mathrm{p}$ to $\mathrm{n}$ doping gradient through the junction. The data on the right illustrates the rectifying effect of each junction, while the data on the left illustrates the improved memory performance. Note that absolute current values are utilized to read the status of the memory, and that the switching amplitude is at least a factor of 3 for all bits. 
A significant advantage of the crosspoint architecture is that, for a given patterning resolution, it will scale to higher bit densities than any other type of electronically addressable memory, a point which we will utilize below. However, crosspoint architectures also have challenges. Note that for the data of Fig. 9, it is the relative, rather than the absolute current values of the individual bits, that were utilized to determine the state of an individual junction. One of the key issues of a crosspoint architecture is that every junction is electronically coupled to every other junction, and thus changing the status of a few bits actually can affect the absolute current values through all of the other bits. One solution is to construct diodes at each of the junctions. Fig. 10 represents a second generation memory circuit in which we constructed a diode in series with each crosspoint junction by establishing a $\mathrm{p}-\mathrm{n}$ doping gradient through the underlying poly-Si wire. By thinning the poly-Si wire (using reactive ion etching processes) in the regions between the crossed wires, it was possible to establish current pathways through the junction that passed through a $\mathrm{p}-\mathrm{n}$ diode prior to passing through the molecular junction. Characteristic data from that memory circuit, are shown in Fig. 10. Note that this memory has a significantly improved performance - the signal level between a ' 1 ' and a ' 0 ' is recorded as an absolute current value, and the ability to discriminate between a ' 1 ' and a ' 0 ' is significantly improved. This diode concept also decreases the energy requirements of such a memory, and enables somewhat more flexible operation. In fact, this particular circuit (and the data string shown in Fig. 10) was configured to act as a complementary input XOR wired-logic gate. The details of wired-logic gates may be found elsewhere. ${ }^{29}$

Molecular electronic memory and (wired) logic circuits, based upon bistable molecular mechanical systems, can clearly be constructed using conventional lithographic patterning methods. For example, the data presented in Figs. 9 and 10 was from memories that were patterned using a combination of optical lithography and metals deposition through evaporation shadow masks. However, one of the key advantages to molecular electronics is that it should scale to near molecular dimensions. The challenge of constructing circuits at such dimensions is not unique to the field of molecular electronics. In fact, pushing the limits of patterning methods continues to be a key challenge for much of the electronics industry. The highest resolution patterning method is electron beam lithography (EBL), which can pattern silicon structures with dimensions of just a few nanometers (although with considerable structure-to-structure variability). However, EBL is severely limited in terms of the density of structures that can be patterned. For example, high aspect ratio silicon wires can be patterned at a center-to-center separation distance (pitch) of about 60-70 nm. ${ }^{30}$ Thus, the development of methods for fabricating electronic circuits with molecular scale feature sizes and pitches constitute a significant challenge. We turn now to a discussion of ultra-high density electronic circuitry.

A couple of years ago we reported on the superlattice nanowire pattern transfer, or SNAP, method for constructing ultra-high density nanowire and crosspoint circuits. ${ }^{31}$ This patterning method, which translates the atomic-level control over layer thicknesses that can be achieved within $\mathrm{GaAs} / \mathrm{Al}_{x} \mathrm{Ga}_{(1-x)} \mathrm{As}$ superlattices into atomic-level control over widths and pitches of nanowires, has been demonstrated to produce nanowire circuits at a density and with a level of fidelity that far exceeds any competing approach. For example, $8 \mathrm{~nm}$ wide nanowires patterned at a pitch of $16 \mathrm{~nm}$ and with lengths of hundreds of micrometres to a few millimetres have been fabricated. ${ }^{31}$ A further advantage of the SNAP method is that it can be utilized to fabricate nanowires of virtually any metal or semiconducting material, including complex structures, such as nanowires constructed of multiple layers of material. We subsequently developed non-destructive doping and reaction ion etching processes for silicon nanowire fabrication. By coupling those processes with the SNAP patterning method, we were able to fabricate silicon nanowires with bulk-like conductivity characteristics. ${ }^{32}$ This is significant because of the extreme sensitivity of the electronic properties of one-dimensional conductors towards even very small numbers of defects. Finally, working with colleagues at Hewlett Packard, we have recently reported upon methods for replicating these structures in high throughput. ${ }^{33}$ This provides us with the key step towards having a realistic manufacturing method that can approach molecular dimensions in scale.

In Fig. 12 we present scanning electron micrographs of a 4.5 kbit memory. This memory was wired up so that many of the wires were connected to one another to make the testing simpler. Only 64 effective bits, with each effective bit containing between 4 and 200 actual junctions, were individually probed. In the inset of Fig. 12 we present data measured from a similarly fabricated circuit. The data represents the switching responses from several of the smallest of those bits (four 


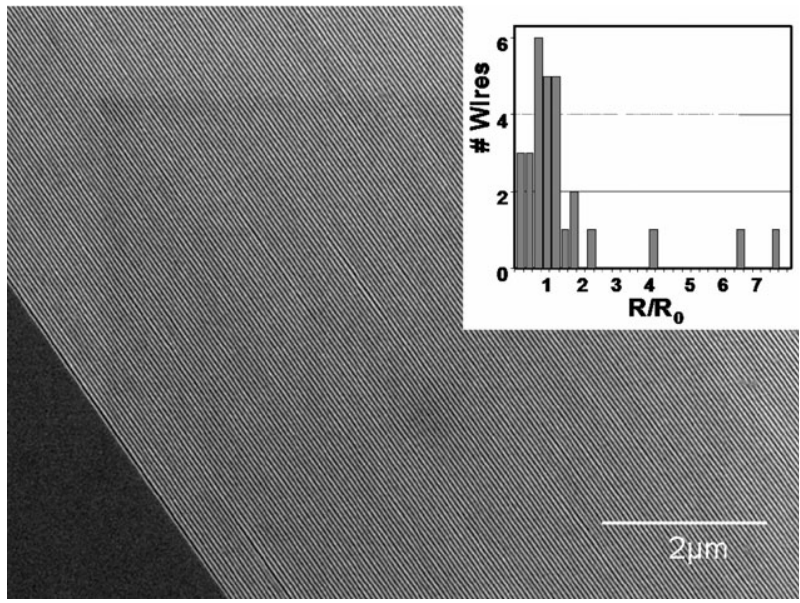

Fig. 11 SNAP-patterned silicon nanowires. Each nanowire is $15 \mathrm{~nm}$ wide by $30 \mathrm{~nm}$ tall, patterned at $34 \mathrm{~nm}$ pitch, and approximately $3 \mathrm{~mm}$ long. These wires were produced on a $\mathrm{Si}(111)$ on insulator (SOI) substrate. The electrical characteristics of a statistical number of these nanowires are shown in the top inset. $R_{0}$ is the calculated resistivity of a nanowire based upon knowledge of the doping level of the SOI wafer. $R$ is the experimentally measured value. Thus these wires are essentially defect-free as evidenced by the fact that their electrical properties are as good as they can possibly be.

or five junctions each). About half of these devices worked, but the device performance is not too dissimilar to what was observed for the data presented in Fig. 11. Each junction in this circuit is about $10 \mathrm{~nm} \times 40 \mathrm{~nm}$ wide, and so contains on the order of 300 molecules. The memory circuits shown in Figs. 9 and 10 each contained on the order of $10^{8}$ molecules or so.

Thus, the circuit of Fig. 12 provides a compelling demonstration that these bits will scale well to reasonably small numbers of molecules. We are currently fabricating and testing 160,000 bit memory circuits fabricated using SNAP (Si) nanowires as the bottom electrodes and SNAP metallic wires as the top electrodes. Those circuits are at a density of $10^{11}$ bits $\mathrm{cm}^{-2}$, and represent a bit density that would correlate to around the year 2020 according to standard Moore's law protocols.

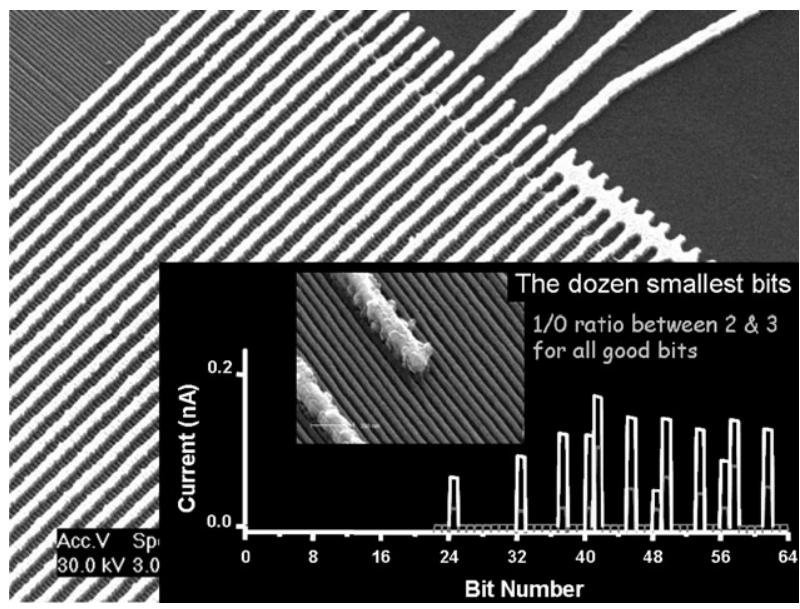

Fig. 12 (Background image) Electron micrograph of a $4.5 \mathrm{kbit}$ molecular electronic memory circuit consisting of $10 \mathrm{~nm}$ wide Si bottom electrodes, $40 \mathrm{~nm}$ wide Ti/Al top electrodes, and a layer of bistable $\left(\mathrm{RTTF}^{4+}\right) \mathrm{rotaxanes}^{\mathrm{N}}$ sandwiched in between. Each junction contains $~ 300$ molecular switches. The inset shows electrical contacts to the Si nanowires, as patterned by electron-beam lithography. Note that each contact touches three to four nanowires. Also shown is the performance of the smallest 13 bits (out of $\sim 24$ such bits). 


\section{Interconnecting ultra-high density molecular electronics circuits}

One final issue that we have addressed involves interconnect architectures that can fully take advantage of ultra-dense electronic circuitry. ${ }^{34}$ Note that in Fig. 12, EBL is not sufficiently highresolution to enable the addressing of individual nanowires. Architectural concepts for meeting the challenge of electrically addressing (demultiplexing) individual nanowires that are patterned at sublithographic densities should satisfy three criteria. First, the demultiplexer architecture must bridge from the micrometre or submicrometre dimensions achievable through lithography to the fewnanometre dimensions achievable through alternative patterning methods.

Second, the architecture should allow for the addressing of many nanowires with a few large wires. Third, the manufacture of the multiplexer should be tolerant of fabrication defects.

Proposed demultiplexer architectures ${ }^{35,36}$ have been based upon combining the concepts of crossbars $^{37}$ (lithographically patterned demultiplexing address wires crossing the nanowires) with multi-input binary tree demultiplexers. ${ }^{38}$ Binary trees, by their very nature, exhibit order $2 \log _{2}(N)$ scaling, where $N$ is the number of nanowires, and $2 \log _{2}(N)$ is the number of the large demultiplexing wires utilized to address the nanowires. An example of a binary tree demultiplexer is shown in Fig. 13.

Nanowire assemblies are often characterized by some randomness in organization, as well as defects, such as broken or non-conducting nanowires. To compensate, the proposed demultiplexing schemes have contained a certain amount of redundancy (extra wires). The major cost of this redundancy is that the use of additional address wires implies that certain nanowire addresses will be redundant or non-active, and circuit testing must be carried out to determine the good addresses, and memory must be devoted to storing those addresses. Kuekes and Williams ${ }^{35}$ described a diodeor resistor-based decoder that utilizes $5 \times \log _{2}(N)$ large microscale address wires crossing an array of $N$ nanowires. DeHon, Lincoln and Savage ${ }^{36}$ described an architecture that utilizes no more than $\left[2.2 \times \log _{2}(N)\right]+11$ address wires. Their scheme is based upon the field-effect gating of nanowires by the demultiplexer, and requires control over the doping profile along the axial dimension of the nanowires. Such nanowires have been realized experimentally, ${ }^{39-41}$ and Lieber's group has used them to demonstrate a demultiplexer that bridges fabrication methods (i.e. self-assembly versus lithographic patterning), but not length scales. ${ }^{42}$ Both of these architectures are based upon placing controllable regions on the surface of the nanowires. An individual nanowire, which is initially in the non-conducting state, will conduct only when all of the control regions are field- or voltageaddressed-i.e. it is the logical equivalent of a multi-input AND gate.

Our approach takes full advantage of the perfect fabrication characteristics of the SNAP patterning method, and it takes full consideration of both the full possibilities and limitations of conventional patterning methods. Fig. 14 illustrates our concept. We have demonstrated this approach, which is described in significant detail elsewhere, ${ }^{34}$ does work for selectively addressing

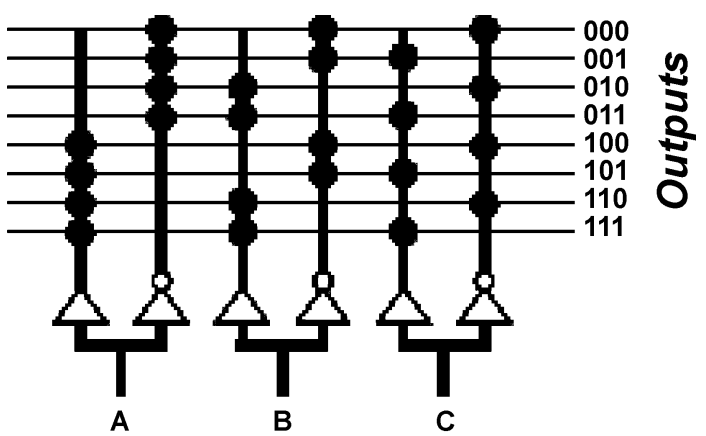

\section{Inputs}

Fig. 13 A binary tree demultiplexer. The operation of this circuit may be understood by considering each of the eight horizontal wires as having an output that has the truth table of a three-input AND gate, with inputs defined by $\mathrm{A}, \mathrm{B}$ and $\mathrm{C}$. There are eight possible input addresses, each defining one of the eight horizontal wires. Note that $2^{3}$ outputs are defined by three input wire pairs, so that the scaling is $2 \log _{2}(N)$ for $N$ outputs. 


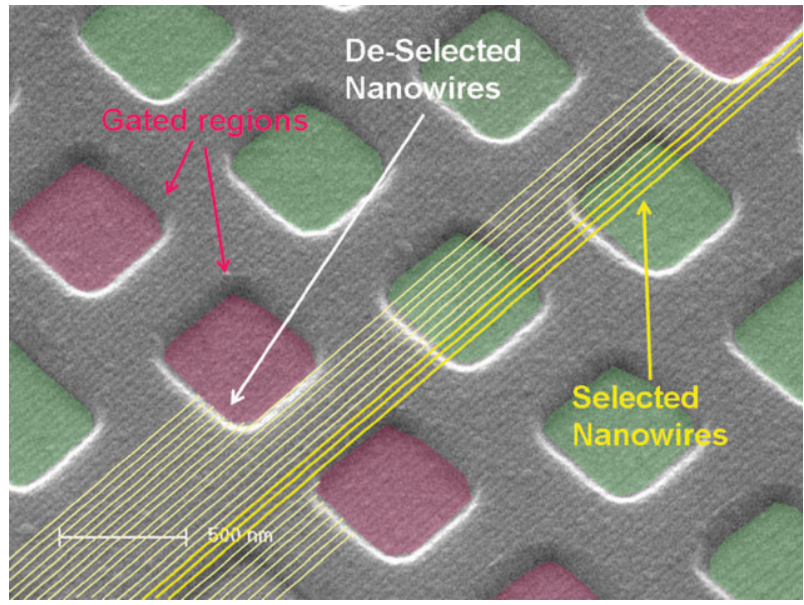

Fig. 14 The nanowire demultiplexer concept, shown as a drawing on top of an electron micrograph. The outline of SNAP-fabricated Si nanowires can be seen as the diagonal strips proceeding from bottom left to top right. The trenches represent regions in which a high- $k$ dielectric is deposited on the nanowire surfaces. Application of a high voltage in certain regions (shaded red) decreases the conductivity of the underlying nanowires, through a field effect. A few nanowires are highlighted in yellow. Only three nanowires pass through this part of the demultiplexer circuit in the high-conductance state, and so are selected. Note that the EBL pattern demux structure is patterned at a much larger feature size and pitch than are the underlying nanowires.

nanowires, although significant challenges remain before it can be fully exploited for demultiplexing large and ultra-dense Si nanowire arrays. Many of those challenges relate to achieving molecular or atomic control over the nanowire/dielectric interfaces within the demultiplexer structure.

\section{Conclusions}

In this Discussion we have tried to present some of the key aspects that are required for building a science and technology that is molecular electronics. However we have also just touched at the surface, with only a few of the key issues described, and even those descriptions are not in depth.

One such issue relates to the ability, through thermodynamic and kinetics measurements, to establish a feedback loop between molecular synthesis and the performance of switching molecular electronic devices. Other groups are taking different routes towards establishing similar relationships. However, a second issue, not covered in this paper, relates to achieving control over the molecule-electrode interface, and how to design or choose electrode materials that can take full advantage of molecular properties. Furthermore, the key circuit demonstrations discussed herein are the result of simply copying applications that we already know exist, such as memory and logic. Such existing applications may be the most difficult for a true molecular electronics technology to compete in, but other areas, such as molecular electronics for sensing and responding, mechanical actuation, etc., may be the most promising avenues for real world applications. This is because in certain of those areas, molecular electronics provides a unique and enabling pathway. However, certain of the challenges associated with learning how to manufacture with high precision at the molecular scale are likely to have broader applications than those discussed here. For example, issues of defect tolerant circuitry, ultra-high density patterning, interconnects, etc., are much broader than their applications to molecular electronics. Regardless of what applications eventually emerge, the science that is currently happening is tremendously rich and fun.

\section{Acknowledgements}

This work was funded by the Department of Energy, the DARPA Moletronics Program, and the MARCO Center for Advanced Materials and Devices. We would also like to acknowledge the 
many current and former members of the Stoddart and Heath groups who have contributed to the science described in this article.

\section{References}

1 J. R. Heath, P. J. Kuekes, G. Snider and R. S. Williams, Science, 1998, 280, 1716.

2 R. L. McCreery, Chem. Mater., 2004, 16, 4477-4496.

3 C. P. Collier, G. Mattersteig, Y. Li, E. W. Wong, K. Beverly, J. Sampaio, F. Raymo, J. F. Stoddart and J. R. Heath, Science, 2000, 289, 1172-75.

4 L. Sheeney-Haj-Ichia and I. Willner, J. Phys. Chem. B, 2002, 106, 13094-13097.

5 Y. Luo, C. P. Collier, K. Nielsen, J. Jeppesen, J. Perkins, E. DeIonno, A. Pease, J. F. Stoddart and James R. Heath, ChemPhysChem, 2002, 2002(3), 519.

6 E. Katz, L. Sheeney-Haj-Ichia and I. Willner, Angew. Chem., Int. Ed., 2004, 43, 3292-3300.

7 (a) M. D. Jimenez, C. Dietrich-Buchecker and J.-P. Sauvage, Angew. Chem., 2000, 112, 3422-3425; (b) M. D. Jimenez, C. Dietrich-Buchecker and J.-P. Sauvage, Chem. Commun., 2003, 1613-1616; (c) M. J. Marsella and R. J. Reid, Macromolecules, 1999, 32, 5982-5984; (d) E. W. Taylor, Science, 1993, 261, 35-36.

8 D. W. Steuerman, H.-R. Tseng, A. J. Peters, A. H. Flood, J. O. Jeppesen, K. A. Nielsen, J. F. Stoddart and J. R. Heath, Angew. Chem., 2004, 43, 2-7.

9 (a) A. H. Flood, A. J. Peters, S. A. Vignon, D. W. Steuerman, H.-R. Tseng, S. Kang, J. R. Heath and J. F. Stoddart, Chem. Eur. J., 2004, 10, 6558; (b) J. W. Choi et al., Ground state equilibrium thermodynamics and switching kinetics of bistable [2]rotaxane switches in solution, polymer gels, and molecular electronic devices, Chem. Eur. J., 2005, in press.

10 (a) J. O. Jeppesen, J. Perkins, J. Becher and J. F. Stoddart, Org. Lett., 2000, 2, 2631-2634; (b) J. O. Jeppesen, J. Perkins, J. Becher and J. F. Stoddart, Angew. Chem., Int. Ed., 2001, 40, 1216-1221; (c) J. O. Jeppesen et al., Chem. Eur. J., 2003, 9, 2982-3007; (d) T. Yamamoto, H. R. Tseng, J. F. Stoddart, V. Balzani, A. Credi, F. Marchioni and M. Venturi, Collect. Czech. Chem. Commun., 2003, 68, 1488-1514; (e) H.-R. Tseng et al., Chem. Eur. J., 2004, 10, 155-172; ( $f$ ) S. Kang, S. A. Vignon, H.-R. Tseng and J. F. Stoddart, Chem. Eur. J., 2004, 10, 2555-2564; (g) I. C. Lee, C. W. Frank, T. Yamamoto, H.-R. Tseng, A. H. Flood, J. F. Stoddart and J. O. Jeppesen, Langmuir, 2004, 20, 5809-5828; (h) J. O. Jeppesen, S. Nygaard, S. A. Vignon and J. F. Stoddart, Eur. J. Org. Chem., 2005, 196-220.

11 (a) E. Katz, O. Lioubashevsky and I. Willner, J. Am. Chem. Soc., 2004, 126, 15520-15532; (b) D. Ryan, S. N. Rao, H. Rensmo, D. Fitzmaurice, J. A. Preece, S. Wenger, J. F. Stoddart and N. Zaccheroni, J. Am. Chem. Soc., 2000, 122, 6252-6257; (c) B. Long, K. Nikitin and D. Fitzmaurice, J. Am. Chem. Soc., 2003, 125, $15490-15498$.

12 (a) A. M. Brouwer, C. Frochot, F. G. Gatti, D. A. Leigh, L. Mottier, F. Paolucci, S. Roffia and G. W. H. Wurpel, Science, 2001, 291, 2124-2128; (b) A. Altieri, G. Bottari, F. Dehez, D. A. Leigh, J. K. Y. Wong and F. Zerbetto, Angew. Chem., Int. Ed., 2003, 42, 2296-2300; (c) A. Alteri, F. G. Gatti, E. R. Kay, D. A. Leigh, D. Martel, F. Paolucci, A. M. Z. Slawin and J. K. Y. Wong, J. Am. Chem. Soc., 2003, 125, 8644-8654; (d) F. Cecchet, P. Rudolf, S. Rapino, M. Margotti, F. Paolucci, J. Baggerman, A. M. Brouwer, E. R. Kay, J. K. Y. Wong and D. A. Leigh, J. Phys. Chem. B, 2004, 108, 15192-15199.

13 (a) A. Livoreil, C. O. Dietrichbuchecker and J. P. Sauvage, J. Am. Chem. Soc., 1994, 116, 9399-9400; (b) J. P. Collin, P. Gavina and J. P. Sauvage, New J. Chem., 1997, 21, 525-528; (c) L. Raehm, J. M. Kern and J. P. Sauvage, Chem. Eur. J., 1999, 5, 3310-3317; (d) I. Poleschak, J. M. Kern and J. P. Sauvage, Chem. Commun., 2004, 474-476.

14 D. W. Steuerman, H.-R. Tseng, A. J. Peters, A. H. Flood, J. O. Jeppesen, K. A. Nielsen, J. F. Stoddart and J. R. Heath, Angew. Chem., Int. Ed., 2004, 43, 6486-6491.

15 H.-R. Tseng, D. Wu, N. X. Fang, X. Zhang and J. F. Stoddart, ChemPhysChem, 2004, 5, 111-116.

16 A. H. Flood, A. J. Peters, S. A. Vignon, D. W. Steuerman, H.-R. Tseng, S. Kang, J. R. Heath and J. F. Stoddart, Chem. Eur. J., 2004, 10, 6558-6564.

17 E. E. Polymeropoulos and J. Sagiv, J. Chem. Phys., 1978, 69, 1836.

18 T. Lee, W. Wang, J. F. Klemic, J. L. Zhang, J. Su and M. A. Reed, J. Phys. Chem. B, 2004, 108, 8742.

19 R. M. Metzger, Chem. Rev., 2003, 103, 3803-3834.

20 R. L. Carroll and C. B. Gorman, Angew. Chem., 2002, 114, 4556-4579.

21 G. Maruccio, R. Cingolani and R. Rinaldi, J. Mater. Chem., 2004, 14, 542-554.

22 (a) G. J. Ashwell, J. R. Sambles, A. S. Martin and W. G. Parker, J. Chem. Soc., Chem. Commun., 1990, 1374-1376; (b) A. S. Martin, J. R. Sambles and G. J. Ashwell, Phys. Rev. Lett., 1993, 70, 218-221.

23 G. Ho, J. R. Heath, M. Kondratenko, D. F. Perepichka, K. Arsenault, M. Pézolet and M. R. Bryce, Chem.Eur. J., 2005, 11, 2914-2922.

24 J. Chen, M. A. Reed, A. M. Rawlett and J. M. Tour, Science, 1999, 286, 1550.

25 N. P. Guisinger, M. E. Greene, R. Basu, A. S. Baluch and M. C. Hersam, Nano Lett., 2004, 4(1), 55-59.

26 A. R. Pease, J. Jeppesen, J. F. Stoddart, Yi Luo, C. P. Collier and J. R. Heath, Acc. Chem. Res., 2001, 34, 433.

27 A. H. Flood, J. F. Stoddart, D. W. Steuerman and J. R. Heath, Science, 2004, 306, 2055-2056.

28 E. Katz, L. O. Shenney-Haj-Ichia and I. Willner, Angew. Chem., Int. Ed., 2004, 43, 3292-3300. 
29 C. P. Collier, E. Wong, M. Belohradsky, F. Raymo, J. F. Stoddart, P. J. Kuekes, R. S. Williams and J. R. Heath, Science, 1999, 285, 391.

30 C. Vieu et al., Appl. Surf. Sci., 2000, 164, 111.

31 N. Melosh, A. Boukai, F. Diana, B. Geradot, A. Badolato, P. Petroff and J. R. Heath, Science, 2003, 300, 112.

32 R. Beckman, E. Johnston-Halperin, Y. Luo, N. Melosh, J. Green and J. R. Heath, J. Appl. Phys., 2004, 96(10), 5921-5923.

33 G. Y. Jung, W. Wu, Z. Yu, S. Y. Wang, Z. Li, R. S. Williams, E. Johnston-Halperin, J. E. Green, A. Boukai, Y. Bunimovich and J. R. Heath, 2005, submitted.

34 R. Beckman, E. Johnston-Halperin, Y. Luo, J. E. Green and J. R. Heath, Science, 2005, DOI: 10.1126/ science. 1114757.

35 P. J. Kuekes and R. S. Williams US Pat. 6,256,767 (3 July 2001).

36 A. DeHon, P. Lincoln and J. Savage, IEEE Trans. Nanotechnol., 2003, 2, 165.

37 J. Heath, P. Kuekes, G. Snider and R. S. Williams, Science, 1998, 280, 1716.

38 R. P. Feynman, Lectures in Computation, ed. A. J. G. Hey and R. W. Allen, Addison-Wesley, Menlo Park, CA, 1996.

39 M. S. Gudiksen et al., Nature, 2002, 415, 617.

40 Y. Wu, R. Fan and P. Yang, Nano Lett., 2002, 2, 83-86.

41 M. T. Björk et al., Nano Lett., 2002, 2, 87-89.

42 Z. Zhong, Science, 2003, 302, 1377. 